\title{
Top Management Team Power Distribution and its Effect
}

\author{
Peng Xie ${ }^{\mathrm{a}}$, Lu Ma ${ }^{\mathrm{b}}$ \\ School of management, Guangxi University of Science and Technology, Liuzhou 545006, China. \\ a pengxie07@163.com, ${ }^{\mathrm{b}}$ malu6655@163.com
}

Keywords: top management team, power distribution, effect.

\begin{abstract}
The intensifying competition in the market leads innovation to become a new weapon for enterprises to improve their market competitiveness. As an important decision-maker and executor of corporate strategy, the top management team (TMT) is the core group to decide on organizational outcomes, such as strategic choices and performances, etc. Power is one of the most crucial parts of TMT. Researchers in the field of strategic management have reported that TMT power disparity has significant impacts on top managers' cognition, emotion, decision processes and firm performance. This paper reviews related literature, which provides deep insights on the influence of power of TMT. Some argued that TMT with unequal power distribution would promote the development of the firm, while others found that equal power distribution would make a positive impact on the team. Accordingly, this paper conducts a literature review focusing on the definitions, influencing factors and effects of top management team power distribution. Finally, it points out the existing drawbacks and some future research directions.
\end{abstract}

\section{Introduction}

In recent years, the process of global economic integration has accelerated significantly, and the Chinese enterprises must face the increasing risk of economic disputes due to the complex environment. Confronted with such a complex and changeable environment, the enterprise must build a highly efficient and integrated executive team. As a core group in the organization, the top management team (TMT) plays a vital role in deciding the direction of business development and obtaining competitive advantages. Since Hambrick brought the "Upper-echelon theory" in 1984, the empirical research of TMT developed very quickly. This theory sees the TMT as a whole and discusses the relationship among the TMT, corporate strategy, and performance. Subsequently, research of TMT was focused on the direct correlations of TMT compositional traits with corporate strategy and performance [1].

As the research going, the relationship between TMT and organizational development is more and more deeply and widely. The influence of TMT power is one of the topics that has drawn more and more attention. In fact, as an important factor influencing the attitude, behavior and working relationship of members, power has always been a delicate topic. Power is the core of the organizational decision-making system, any one of TMT will be disproportionately affected by power during the process of organization. Meanwhile, power has two sides, there can be a positive or negative side in the different organizational environments. Regardless of the balance of power distribution or imbalance in the TMT, it is possible to bring positive or negative effects on the enterprise. Through making a comprehensive view of the study of dynamical and international development, most scholars pay more attention to the unbalanced distribution of power but ignore the research on the balance of power distribution, and there are still differences between the scholars' conclusions $[2,3]$. These conclusions may be reasonable under the specific boundary conditions, but how does the distribution of TMT power be more conducive to the development of the organization? What are the factors that affect the effectiveness of the distribution of TMT power? These questions are increasingly becoming the focus concerned by theory field and practice. Therefore, based on the exposition of the theory of organization hierarchy and power distance theory, this article illustrates the TMT power distribution and its effect, in order to provide reference and guidance for the subsequent theoretical research in this field and the management practice of the TMT. 


\section{Power Distribution of TMT}

Power distribution of TMT means that the enterprise resources within the organization are distributed among the TMT members [4], which affects the formulation and implementation of the business development strategy. To some extent, the power distribution of the TMT may affect the enterprise management and its development more than the power size itself. Cao et al. (2015) pointed out that TMT power distribution is to specifically allocate the power within the organization members [5]. The balance of power distribution corresponds to the collective leadership, while power is unbalance distributed and very few executive team members have most of the power, which corresponds to individual leadership. Mao (2016) also pointed out that TMT power distribution can reflect the size of the power of the members of the TMT, and the power gap is the external manifestation of the imbalance of power distribution [6].

According to the internal structure of the organization, the power model of the TMT can be divided into two different types: horizontal and vertical structures. The horizontal structure mainly reflects the distribution of power among the various departments within the organization, while the vertical structure highlights the differences among the internal members of the team resulting from their different levels. Research shows that horizontal and vertical structures can have different effects on organizations. The former usually leads to organizational inertia, while the latter easily leads to organizational change.

\section{Influence Factors of TMT Power Distribution}

\subsection{Leader Style.}

Leadership style is the behavior pattern exhibited by the leader in the process of managing the enterprise with certain plasticity and individuality. In the Western context, due to the small distance of social power, executives in the enterprise can more easily obtain the authority of the highest authority. However, the Chinese show a relatively large power distance, and the power of TMT members is relatively concentrated. So in Chinese and western business management, there will be differences in the authorization styles in the organization, thus affecting the distribution of power in the TMT.

Specifically, Chinese paternalistic leadership is a form of leadership behavior that manifests in personality, contains authority, fatherly kindness and virtue. It specifically includes three important dimensions: authoritarianism, benevolence, and virtue. Among them, authoritarianism emphasizes absolute authority and implements strict disciplines in organizations. But shared leadership is a dynamic and interactive process within a group and is a team process in which members lead each other. Some researchers believe that, unlike the traditional vertical, top-down leadership model, shared leadership is a horizontal, bottom-up, collective leadership model that is organized internally by employees. This model reflects more fairness for the TMT members, ensures that the distribution of power within the organization is relatively balanced and does not result in excessive concentration of power in the hands of a senior executive.

In short, due to the various leadership styles of the top leaders, different authorization atmospheres will be created within the TMT. If the highest authority is biased toward the collective leadership, the distribution of power within the team will be relatively balanced. Conversely, if the highest authority is biased toward the individual leadership, the power within the team will be concentrated on the hands of a few executives, resulting in an unbalance distribution of TMT power.

\subsection{Compositional Traits of TMT.}

The power distribution of the TMT will be influenced by the team size and social status. These factors make up the characteristics of TMT members, so they can indirectly reflect the special experience, values and personality traits of the members of the executive team. It is usually possible to analyze the composition of the executive team from two perspectives: content and structure. On the view of content analysis, it generally can choose some measurable variables, such as age, gender, started experiencing, to explore whether executive team composition characteristics have an impact on organization. On the perspective of structural analysis, it focuses on the similarity of the individual 
characteristics of senior executives, and studies the influence of the heterogeneity or homogeneity of the executive team on the organization.

Basu (2000) believed that the size of the TMT has its own rationality. On the one hand, if the TMT is large, the distribution of power in the team is relatively dispersed, and the organization can acquire more information and knowledge. On the other hand, if the TMT is small, the power will be concentrated on individual managers, and the views of the team will be easy to unify. Some research suggests found that these two components have a significant correlation with the power distribution within the executive team, through the research on the size of the executive team and the education background. Liu (2009) found that different executive function background, there is an obvious difference on the same thing, and failed to reach a consensus among the member, thus susceptible to small groups, block information exchanges and cooperation between members. These small groups can easily lead to the emergence of "office politics", which can influence the distribution of power within the executive team.

\section{The Effect Mechanism of TMT Power Distribution}

\subsection{The Consequence of Balance of Power Distribution in TMT.}

When the power of the TMT is balanced, it has a two-sided impact on individuals and organizations.

On the positive side, the balanced distribution of power among the senior management team advocates the collective behavior of the collective leadership, which is conducive to the formation and enhancement of team cohesion. This collective leadership can promote information sharing, division of labor and strategic decision-making among internal members, and ensure that team members have the same attitude towards strategic choices, which helps organizations grasp key opportunities to adjust strategy, and obtain a continuous competitive advantage. Whitson (2013) found that when a person is empowered, he will become more focused on the company's goals, which is conducive to consensus [7].

On the negative side, if each executive has the opportunity to express their views, there may be slow and endless discussions in the team, which may lead to the organization losing the opportunity to formulate and implement the strategy and seriously harming the interests of the company. Because the power between members is equal, problems such as unclear division of responsibilities among internal members of the senior management team and unclear division of labor are easily encountered, which hinders the development of the organization. Chun and Choi (2014) also found that the senior members of the organization are often not satisfied with having equal rights, they will strive for higher power, and then induce identity conflicts among members [8]. Previous studies have shown that conflicting identity among team members can hinder the cooperation and information sharing between team members [9], thereby reducing the ability of executive team members to make decisions.

\subsection{The Consequence of Unbalance of Power Distribution in TMT.}

When the power of the TMT is unbalanced, it also has a two-sided impact on individuals and organizations.

From a positive perspective, Willer (2014) pointed out that high-powered people usually take a positive attitude towards work and have a higher sense of organizational identity and work mission [10]. This can allow individuals to exercise restraint in irresponsible impulses at work and thus demonstrate a very high work commitment. Greve and Mitsuhashi (2007) showed that if the leadership is concentrated in individual executives in the senior management team, not only unnecessary disputes can be avoided, but also the time for information exchange between members can be saved [11]. In the case of uneven distribution of power, senior executives will use their privileges to establish team rules to better regulate the behavior of senior executives. Meyer et al. (2013) found that differences in power among members of the senior management team can help organizations establish team norms [12], thereby attracting constructive criticism for strategic decisions. 
From a negative perspective, the unequal distribution of the power of the members of an organization can easily increase the perception of unfairness and affect job satisfaction of employees. Consequently, it hinders the realization of organizational goals [13]. Communication between teams plays a key role in promoting high-level organizational performance. Nevertheless, unbalanced power among members of the TMT will hinder the exchange of information within the team and cause problems in the communication mechanism among internal members, thus affecting the overall high the process of managing the team. Pantel and Cooper (2014) found that the low-power executives tend not to actively participate in the management process of the team and remain silent and participate in the management of the enterprise passively [14]. In addition, the study of social psychology points out that the subjective psychological experience brought by power can influence the leadership in the team environment. Galinsky et al. (2008) found that having power often leads to individuals becoming overconfident with their own thoughts, becoming more focused on themselves and ignoring others' thoughts [15]. See et al. (2011) further confirmed that power will lead to changes in subjective perceptions of senior executives and would not listen to others [16].

\section{Conclusions and Future Perspective}

The earliest research of the TMT focused on the influence of demographic characteristics on the organization. The perspective of the subsequent research began to focus on the process of the team, and the relevant research results were also more abundant. As scholars continue to deepen their research on the TMT, the perspectives and concerns of the study have also begun to increase. In these studies, TMT power distribution is an important research topic. Although the research on the power distribution of TMT has achieved some good results, there are still some research deficiencies in the study of this topic, and it still needs to be taken more into consideration.

First, the concept of executive team power needs to be clarified and the tools for measuring the distribution of power still need to be developed. In the existing research, different areas have different definitions of the concept of power, and there is still a lack of systematic integration of the concept of power in the management community. At present, the definition of the concept of power by scholars is mostly from the static point of view, lack of dynamic manifestation, which makes it difficult to reflect the real connotation of the power of the senior management team.

Second, research on what is the influencing factor of the power distribution of the TMT and what effect the TMT power distribution will have on individuals and organizations is still not thorough enough. What factors will affect the distribution of power, what role does power play in the TMT, and whether it will have different effects on the organization through different channels, this issues have yet to be expected to be researched in detail.

Thirdly, there are fewer empirical studies in different social and cultural backgrounds. Current research on the distribution of the executive power is mainly under the western mainstream culture background and values, research is in the oriental culture situation still needs further exploration effectively, especially in China's social and cultural context to explore how executive team power distribution is more reasonable and effective, great research value.

\section{References}

[1]. Barkema H G., Shvyrkov O. Does top management team diversity promote or hamper foreign expansion? Strategic Management Journal. Vol. 28 (2007) No.7, p. 663-680.

[2]. Anderson C., Brown, C E. The functions and dysfunctions of hierarchy. Research in Organizational Behavior. Vol. 30 (2010) No. 1, p. 55-89.

[3]. Wang D., Waldman D A., Zhang Z. A meta-analysis of shared leadership and team effectiveness. Journal of Applied Psychology. Vol. 99 (2014) No.2, p. 181-198.

[4]. Magee J C., Galinsky A D. Social hierarchy: the self-reinforcing nature of power and status. Academy of Management Annals. Vol. 2 (2008) No. 1, p. 351-398. 
[5]. Cao J., Yang B., Yang B Y. Top management team power distribution and firm performance: evidence from listed firms in shanghai and shenzhen stock exchange. Science of Science and Management of S \& T. Vol. 36 (2015) No. 7, p. 135-145.

[6]. Mao X S. A literature review and future perspective on power distribution of top management team. Finance Research.Vol. 1 (2016) No. 2, p. 52-60.

[7]. Whitson J A., Liljenquist K A., Galinsky A D., et al. The blind leading: power reduces awareness of constraints. Journal of Experimental Social Psychology. Vol. 49 (2013) No. 7, p. 579-582.

[8]. Chun J S., Choi J N. Members` needs, intragroup conflict, and group performance. Journal of Applied Psychology.Vol. 99 (2014) No. 2, p. 437-450.

[9]. Bendersky C., Hays N A. Status conflict in groups. Organization Science, Vol. 23 (2012) No. 2, p. 323-340.

[10]. Willer R. Groups Reward Individual Sacrifice: The status solution to the collective action problem[J]. American Sociological Review. Vol. 74 (2014) No. 3, p. 23-43.

[11]. Greve H R., Mitsuhashi H. Power and glory: concentrated power in top management teams. Organization Studies. Vol. 28 (2007) No. 8, p. 1197-1221.

[12]. Meyer B., Glenz A. Team faultline measures: a computational comparison and a new approach to multiple subgroups. Organizational Research Methods. Vol. 16 (2013) No. 3, p. 393424.

[13]. Kennedy, J A., Anderson, C. Micropolitics: a new model of status hierarchies in teams. Research on Managing Groups and Teams. Vol. 15 (2012) No. 1, p. 49-80.

[14]. Patel P C., Cooper D. Structural power equality between family and non-family TMT members and the performance of family firms. Academy of Management Journal. Vol. 57 (2014) No. 6, p. 1624-1649.

[15]. Galinsky A D., Magee J C., Gruenfeld D H., et al. Power reduces the press of the situation: implications for creativity, conformity, and dissonance. Journal of Personality \& Social Psychology. Vol. 95 (2008) No. 6, p. 1450-66.

[16]. See K E., Morrison E W., Rothman N B., et al. The detrimental effects of power on confidence, advice taking, and accuracy. Organizational Behavior and Human Decision Processes, Vol. 116 (2011) No. 2, p. 272-285. 